\title{
Conhecimento, atitudes e práticas dos profissionais de saúde sobre os cuidados paliativos a pacientes oncológicos
}

\author{
Knowledge, attitudes and practices of health professionals about palliative care to cancer \\ patients
}
Conocimiento, actitudes y prácticas de los profesionales de salud sobre los cuidados paliativos a pacientes oncológicos

\begin{abstract}
Silmara de Oliveira Silva ${ }^{1 *}$, Suely Arruda Vidal ${ }^{1}$, Erika Penha Carvalho Oliveira ${ }^{1}$, Gilvania Smith da Nóbrega Morais², Lorena Sampaio Almeida ${ }^{3}$, Keityane Leacarla Bezerra da Silva ${ }^{1}$.
\end{abstract}

\section{RESUMO}

Objetivo: Verificar o conhecimento, atitudes e práticas (CAP) dos profissionais de saúde em relação aos cuidados paliativos a pacientes oncológicos. Métodos: Pesquisa quantitativa com delineamento de corte transversal do tipo CAP, realizada no Instituto de Medicina Integral Professor Fernando Figueira (IMIP) no período de Março de 2016 à fevereiro de 2017. Resultados: Participaram do estudo 57 enfermeiros e 43 médicos. Apenas $9 \%$ dos enfermeiros possuem especialização na área de oncologia. Em relação aos fatores que influenciam a integração dos cuidados paliativos aos pacientes oncológicos foram citados o padrão cultural, formação curativista, medo e pressão familiar. Na prática clínica os médicos oncologistas encaminham poucos pacientes por mês ( 5 a 10) para acompanhamento em ambulatório com os paliativistas. Conclusão: Evidencia-se a necessidade de capacitação dos profissionais, principalmente os enfermeiros que não possuem especialização na área, e o desenvolvimento de estratégias que contribua para aplicação do conhecimento e atitudes na prática diária.

Palavras-chave: Cuidados paliativos, Oncologia, Conhecimentos, Atitudes e Prática em Saúde.

\begin{abstract}
Objective: To assess the knowledge, attitudes and practices (CAP) of health professionals in relation to palliative care to cancer patients. Methods: It is a quantitative research with a cross section of the type CAP held at the Integral Medicine Institute Professor Fernando Figueira (IMIP) in the period from March 2016 to February 2017. Results: 57 nurses participated in the study and 43 physicians. Only $9 \%$ of nurses have expertise in the area of Oncology. In relation to the factors that influence the integration of palliative care to cancer patients there were referred to the cultural standard, care training, fear and family pressure. In clinical practice the doctors forward a few patients a month (5 to 10) for monitoring as an outpatient with the caregivers. Conclusion: It is evidenced the need for training of professionals, especially nurses who do not have expertise in the area, and the development of strategies that contribute to knowledge and attitudes in daily practice.
\end{abstract}

Key words: Palliative Care, Oncology, Knowledge, Attitudes and Practice in Health.

\footnotetext{
${ }^{1}$ Instituto de Medicina Integral Prof. Fernando Figueira (IMIP), Recife-PE.

*E-mail: silmaraolyveira@gmail.com

2 Universidade Federal de Campina Grande (UFCG), Campina Grande-PB

${ }^{3}$ Centro Universitário Tiradentes (UNIT), Maceió-AL.
}

SUBMETIDO EM: 1/2019

ACEITO EM: 2/2019

PUBLICADO EM: 4/2019

REAS/EJCH | Vol. 11 (9) | e369 | DOI: https://doi.org/10.25248/reas.e369.2019 Página 1 de 8 


\section{RESUMEN}

Objetivo: Verificar el conocimiento, actitudes y prácticas (CAP) de los profesionales de salud en relación a los cuidados paliativos a pacientes oncológicos. Métodos: Investigación cuantitativa con delineamiento de corte transversal del tipo CAP, realizada en el Instituto de Medicina Integral Profesor Fernando Figueira (IMIP) en el período de marzo de 2016 a febrero de 2017. Resultados: Participaron del estudio 57 enfermeros y 43 médicos. Sólo el $9 \%$ de los enfermeros tienen especialización en el área de oncología. En relación a los factores que influencian la integración de los cuidados paliativos a los pacientes oncológicos, fueron citados el patrón cultural, formación curativista, miedo y presión familiar. En la práctica clínica los médicos oncólogos encaminan pocos pacientes por mes (5 a 10) para acompañamiento en ambulatorio con los paliativistas. Conclusión: Se evidencia la necesidad de capacitación de los profesionales, principalmente los enfermeros que no poseen especialización en el área, y el desarrollo de estrategias que contribuyan a la aplicación del conocimiento y actitudes en la práctica diaria.

Palabras clave: Cuidados paliativos, Oncología, Conocimientos, Actitudes y Práctica en Salud.

\section{INTRODUÇÃO}

A contextualização da concepção dos cuidados paliativos na história data desde os primórdios da era cristã, através da existência das hospedarias, que acolhiam pessoas que necessitavam de cuidado, transmitindo, mesmo que de forma primitiva, proteção, alívio do sofrimento e atenção. Entretanto, a concepção de cuidados paliativos foi definida pela primeira vez em 1990 pela Organização Mundial de Saúde (OMS), sendo revisada em 2002, incluindo a família na dimensão do cuidar, valorizando a promoção da qualidade de vida para pacientes que estão com doenças que ameacem a continuidade da vida e considerando as dimensões física, psicossocial e espiritual, além de perdurar até o luto (HERMES HR e LAMARCA ICA, 2013).

Vale ressaltar que na difusão da filosofia dos cuidados paliativos, destaca-se Cecily Saunders, a qual foi responsável por incentivar e consolidar uma nova forma de transmitir o cuidado, ressaltando o controle da dor e permitindo o encontro das dimensões psicológicas, social e espiritual dos pacientes e suas famílias, contribuindo para o fortalecimento e consolidação do Movimento do Hospice Moderno e propagação dos cuidados paliativos (CHAVES JHB, et al., 2011).

A prática destes cuidados deve ser proporcionada desde o diagnóstico inicial de uma doença grave, incurável e progressiva que ameace a continuidade da vida. Insere-se nessa modalidade de cuidar os pacientes acometidos pelo câncer que necessitam de uma assistência que contemple sua qualidade de vida, aliviando a dor e sintomas que podem ser controlados, além da atividade interdisciplinar de diferentes profissionais que atuam para promover conforto ao paciente e familiares (SILVA AF, et al., 2015; KASSA H, et al., 2014).

Todavia, a referenciação aos cuidados paliativos tem sido fornecida de forma tardia, postergando o trabalho em conjunto com a oncologia para controle de sintomas e qualidade de vida associado com a melhor terapêutica definida para cada indivíduo. $O$ encaminhamento para serviços de cuidados paliativos ocorre no curso da doença, em média de 30 a 60 dias antes da morte. Dessa forma, é preciso intensificar ações que garantam ao paciente os cuidados de suporte adequado para suas necessidades e, que aconteça a integração dos cuidados paliativos de forma precoce contribuindo para o aumento da sobrevida dos pacientes (CRUZ ACVG, 2013).

No Brasil, a abordagem dos cuidados paliativos ainda é incipiente, sendo necessário os profissionais que atuam nos centros de saúde realizarem capacitações que proporcionem maior conhecimento e oportunize a inserção dos cuidados paliativos na prática clínica. A integração dos cuidados paliativos não acontece de forma paralela ao diagnóstico inicial de uma doença crônica progressiva sem perspectiva de cura, na maioria das vezes é inserido em estágios terminais e assim, não ocorre de forma integral (SILVA RCF E HORTALE VA, 2006). 
Ressalta-se que não é apenas no Brasil que os cuidados paliativos são incipientes, em geral a prática dos cuidados paliativos está fragilizada principalmente nos países de baixa e média renda, (CONNOR SR E BERMEDO MCS, 2014). De acordo com a Organização Mundial de Saúde (OMS) a cada ano 40 milhões de pessoas necessitam de cuidados paliativos e apenas $14 \%$ recebem esses cuidados. Dessa forma, 0 direcionamento dos pacientes para profissionais capacitados que podem contribuir no alívio da dor, do bem estar e qualidade de vida é prejudicado. A importância de inserir os cuidados paliativos precocemente vai ao encontro dos benefícios para os pacientes, pois estes podem se beneficiar de serviços de referência que possuem práticas integrativas e profissionais especialistas em cuidados paliativos. (OMS/WHO, 2018).

Nessa perspectiva, levando em consideração que em muitos casos os cuidados paliativos são inseridos tardiamente, esse estudo surgiu mediante indagações sobre o que levaria os profissionais adotarem tal prática e, se o conhecimento que os profissionais de saúde possuem em cuidados paliativos corresponde com suas atitudes e práticas.

Sendo assim, mediante a necessidade de realizar um diagnóstico sobre a prática dos cuidados paliativos aos pacientes oncológicos, o estudo tem como objetivo verificar o conhecimento, atitudes e práticas dos profissionais de saúde em relação aos cuidados paliativos a pacientes oncológicos.

\section{METODOLOGIA}

Trata-se de uma pesquisa quantitativa com delineamento de corte transversal e abordagem tipo CAP (Conhecimento, Atitudes e Práticas). Os estudos do tipo CAP visam medir o conhecimento, atitudes e práticas de determinados grupos.

O estudo foi realizado no Instituto de Medicina Integral Professor Fernando Figueira (IMIP) que atua nas áreas de assistência médico-social, ensino, pesquisa e extensão comunitária. É credenciado como CACON (Centro de Alta Complexidade em Oncologia), oferecendo assistência especializada e integral aos pacientes, até Serviço de Assistência Domiciliar (SAD). Atualmente o número de leitos da enfermaria oncológica são 32, e mais 14 leitos para cuidados paliativos. Como instituição formadora, oferece pósgraduação lato senso e strictu senso.

A população do estudo foi composta por médicos e enfermeiros que trabalham na oncologia do IMIP, incluindo os residentes de medicina e enfermagem em cancerologia; do Serviço de Pronto Atendimento (SPA), por representar a porta de acesso a muitos pacientes oncológicos. A amostra foi censitária composta por 112 profissionais mediante o quadro de profissionais da oncologia e SPA.

Como instrumento de coleta de dados foi aplicado um questionário com perguntas fechadas referentes ao conhecimento, atitudes e práticas dos profissionais em relação aos cuidados paliativos aos pacientes oncológicos. Posteriormente, para verificar a prática foram consultados os prontuários de pacientes oncológicos para se observar a data da primeira consulta e a data do encaminhamento aos cuidados paliativos entre aqueles que se encontram no ambulatório em um período de três meses.

Em relação aos critérios de inclusão foram levados em consideração: ser médico ou enfermeiro da oncologia e SPA adulto; estar cursando a residência médica em oncologia, enfermagem em cancerologia e os de residência multiprofissional em cuidados paliativos e saúde do idoso; prontuários de pacientes oncológicos atendidos no ambulatório de oncologia por médicos paliativistas. E como critérios de exclusão: ser residente de outra instituição fazendo rodízio no setor de oncologia; profissionais que estejam em licença maternidade ou tratamento de saúde por um período maior que dois meses na época da coleta.

Foram coletados 100 questionários, devido às férias ou licença maternidade de alguns profissionais, os quais foram digitados, processados e analisados utilizando o software EPI-INFO versão 3.3.2.

Este estudo foi aprovado pelo Comitê de Ética do IMIP CAAE: 55480516.3.0000.5201, seguiu as normas delimitadas pela Resolução 466/2012 do Conselho Nacional de Saúde, especialmente no que concerne ao Consentimento Livre e Esclarecido, anonimato e sigilo das informações. A participação foi voluntária, não implicando em ônus ou bônus a seus participantes, estando livres para se retirarem do estudo, em qualquer momento que houvesse algum tipo de prejuízo aos próprios. 


\section{RESULTADOS}

Fizeram parte do estudo 100 profissionais da saúde, sendo 57 enfermeiros e 43 médicos, assim distribuídos: 47 enfermeiros da oncologia e SPA, 36 médicos da oncologia e SPA; residentes: sete de medicina (oncologia), dez de enfermagem (sete em cancerologia e três em cuidados paliativos e saúde do idoso) e a maioria do sexo feminino (76\%). Na tabela 1 segue a caracterização sociodemográfica dos participantes.

Tabela 1 - Caracterização dos participantes da pesquisa quanto à idade, estado civil, tempo de formação, atuação e especialidades. IMIP-PE, 2016.

\begin{tabular}{ccc}
\hline Variáveis & $\mathbf{N = 1 0 0}$ & $\%$ \\
\hline Idade & & \\
\hline $20-30$ & 47 & 47 \\
$31-40$ & 45 & 45 \\
$>40$ & 8 & 8 \\
\hline Estado Civil & & \\
\hline Casado(a)/União Estável & 52 & 52 \\
Solteiro (a) & 48 & 48 \\
\hline Religião & & \\
\hline Católica & 65 & 65 \\
Espírita/evangélica & 25 & 25 \\
Sem religião & 10 & 10 \\
\hline Formação & & \\
Enfermeiro & 57 \\
Médico & 43 & 57 \\
\hline Tempo de Formação & & \\
10 anos & 88 \\
$>10$ anos & 12 & 88 \\
\hline Tempo de Atuação na & & \\
Oncologia/Serviço & & \\
\hline$\leq 5$ & 87 \\
$>5$ & 13 & 13 \\
\hline 5 &
\end{tabular}

Fonte: IMIP-PE, 2016.

Figura 1 - Distribuição das especialidades dos profissionais de enfermagem.

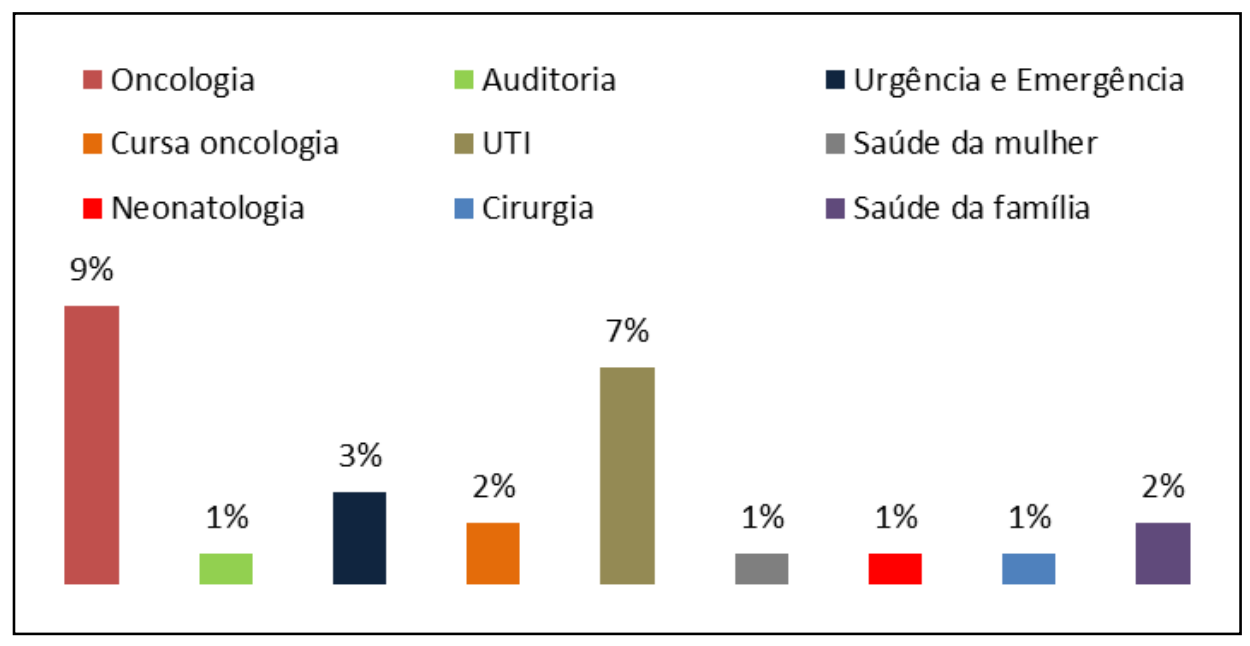

Fonte: questionários - IMIP-PE, 2016. 
Em relação à especialização, 27\% dos profissionais de Enfermagem são especialistas, mas, apenas $9 \%$ são na área de oncologia (Figura 1). Em relação aos médicos, a maioria tem especialização em oncologia, com exceção para os plantonistas do SPA (13\%).

A cerca da definição para cuidados paliativos, um pouco mais da metade $(62 \%)$ relatou que conhecia. Quando questionados sobre a indicação de cuidados paliativos, $89 \%$ responderam que sabiam quais as indicações, associando a doenças graves, avançadas sem perspectiva de tratamento curativo. No que diz respeito à abordagem do tema na graduação a maioria $(66,7 \%)$ relatou que foi insuficiente.

Ainda em relação ao conhecimento, foram expostas aos participantes três afirmações para que respondessem se concordavam ou discordavam. Uma estava relacionada ao aumento da sobrevida com a inserção dos cuidados paliativos precoce, a qual $79 \%$ dos participantes concordaram. As demais afirmações seguem na tabela 2.

Tabela 2 - Afirmações relacionadas ao cuidado paliativo.

\begin{tabular}{|c|c|c|c|}
\hline Afirmações & Concorda & Discorda & Não Sei \\
\hline $\begin{array}{l}\text { 1- O cuidado paliativo precoce aumenta a } \\
\text { sobrevida dos pacientes }\end{array}$ & $79 \%$ & $17 \%$ & $4 \%$ \\
\hline $\begin{array}{l}\text { 2- Os cuidados paliativos só devem ser } \\
\text { fornecidos aos pacientes em fase terminal }\end{array}$ & $13 \%$ & $87 \%$ & - \\
\hline $\begin{array}{l}\text { 3- O cuidado paliativo deve ser iniciado assim } \\
\text { que for estabelecido o diagnóstico de uma } \\
\text { doença que limite a vida do paciente }\end{array}$ & $83 \%$ & $14 \%$ & $3 \%$ \\
\hline
\end{tabular}

Quanto à atitude, os participantes foram questionados sobre o momento de fornecimento de cuidados paliativos ao paciente oncológico metastático, $81 \%$ afirmou que deveria ser no início do tratamento oncológico, todavia $15 \%$ afirmaram que deveria ser durante o processo de terminalidade e $4 \%$ não souberam responder.

Sobre os fatores que influenciam na integração dos cuidados paliativos aos pacientes oncológicos, as respostas variaram de 18,5\% para o padrão cultural a 13,4\% para instituição, como segue na Figura 2:

Figura 2 - Fatores que influenciam a integração dos cuidados paliativos.

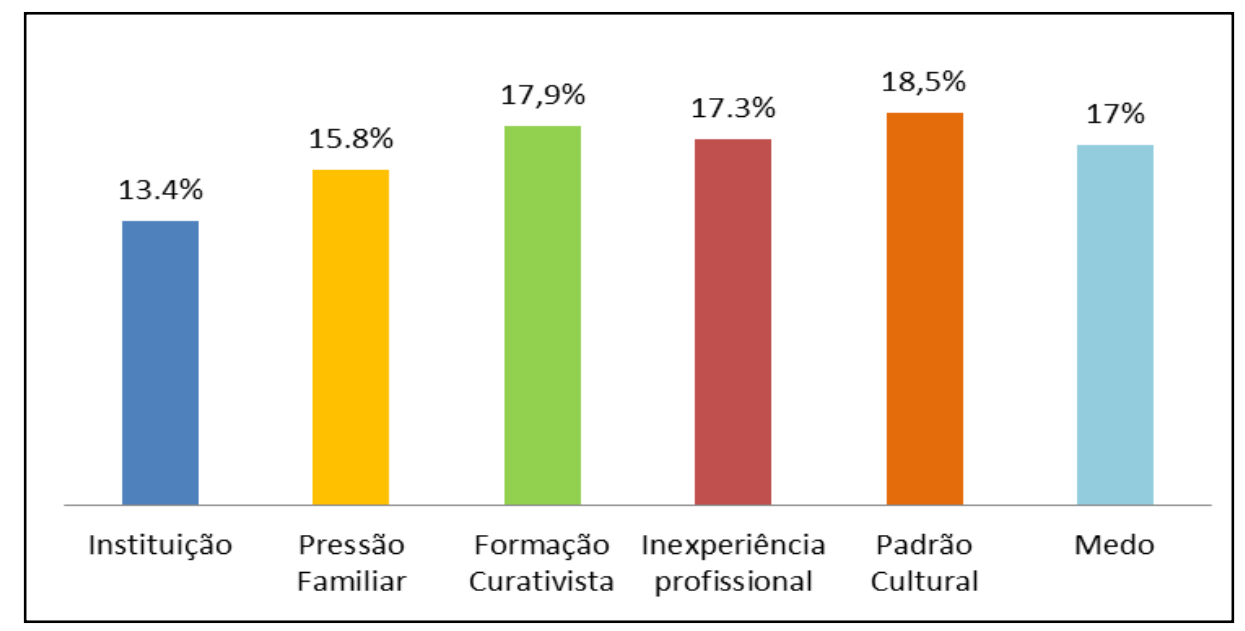

Fonte: IMIP, 2016. 
$\mathrm{Na}$ prática clínica, os médicos oncologistas informaram que encaminham de cinco a 10 (58\%) pacientes por mês para acompanhamento em ambulatório com os paliativistas, contudo, $21 \%$ relataram não encaminhar (Figura 3). Observou-se no prontuário que o tempo médio entre a data de encaminhamento para consulta nos paliativos e a primeira consulta, era 23,6 dias.

Figura 3 - Porcentagem mensal dos pacientes encaminhados para os cuidados paliativos.

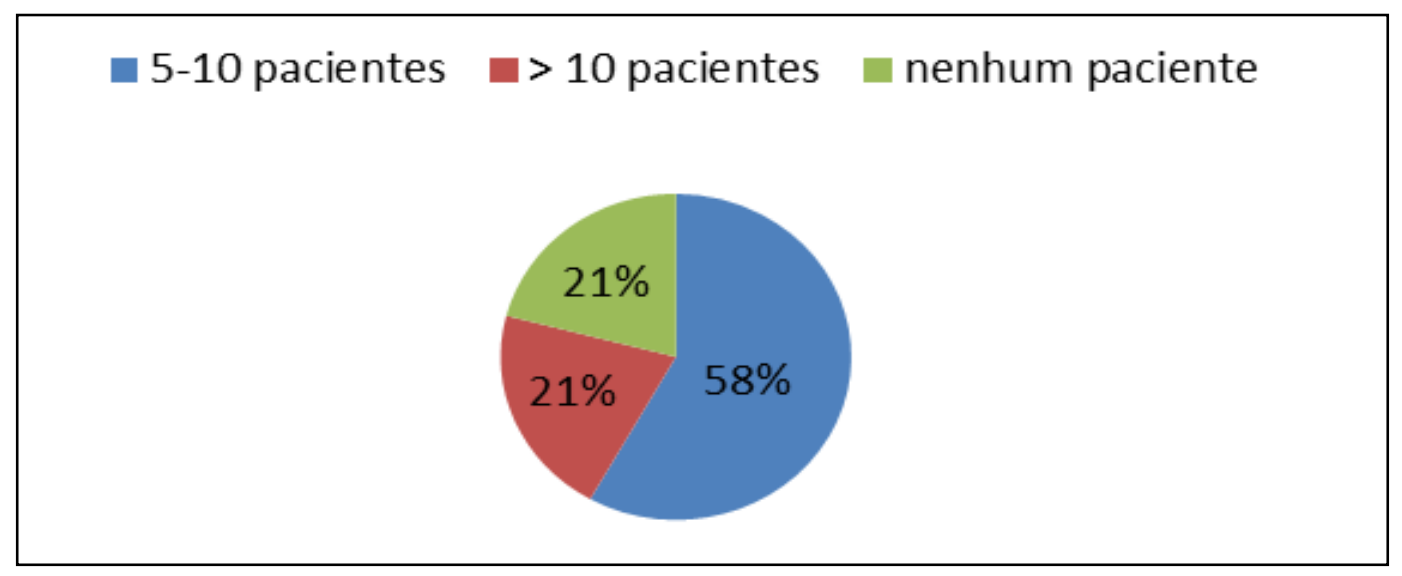

Fonte: IMIP, 2016.

Em relação às práticas não farmacológicas, grande parte $(74 \%)$ recomenda para os pacientes oncológicos atividade física (29\%), acupuntura, yoga, massagem e musicoterapia (5,8\% para cada); alimentação saudável e leitura (3,4\% para cada); lazer (7\%) e práticas religiosas (8\%).

$\mathrm{Na}$ prática clínica também foram questionados sobre a forma de avaliação da dor no paciente oncológico, a maioria referiu o uso de escalas para mensuração (35\%), relato verbal (35\%) ou ambos (22\%) e 8\% relatou não utilizar instrumento para avaliação da dor oncológica.

Os participantes auto avaliaram seu conhecimento em cuidados paliativos como: regular (49\%), insuficiente (43\%) e muito bom (5\%), mas $92 \%$ julgaram ser necessário melhorar o conhecimento.

\section{DISCUSSÃO}

Esse estudo permitiu identificar o conhecimento, as atitudes e práticas dos profissionais de saúde que trabalham numa instituição de referência em oncologia e que também é campo de formação em cuidados paliativos. Observa-se que os profissionais concordam que o cuidado paliativo deveria ser fornecido de forma concomitante com outras terapêuticas, todavia na prática encontram dificuldades para inseri-lo. Esse fato pode ser evidenciado pelo pequeno número de pacientes encaminhados e pelo fato de uma parte dos médicos ainda relatarem não encaminhar, demonstrando que, apesar de ter conhecimento, não aplica na prática. Isso pode ser explicado por fatores sociais que podem interferir nas atitudes na prática clínica conforme seus próprios relatos.

Segundo as diretrizes da American Society of Clinical Oncology (ASCO), os cuidados paliativos devem ser integrados na prática clinica simultaneamente ao tratamento ativo para os pacientes com câncer. Para que isso aconteça, é essencial que os profissionais que trabalham na oncologia associem o conhecimento e suas atitudes para desempenhar um cuidado com excelência (FERREL BR, et al., 2016).

Sob este prisma, o presente estudo aponta o desconhecimento de alguns profissionais sobre a definição dos cuidados paliativos da OMS, sendo necessário investir em capacitações na instituição. Ressalta-se que 
a falta de capacitação em cuidados paliativos pode ocasionar consequências negativas para os profissionais, interferindo consequentemente na assistência aos pacientes (PINHEIRO TRSP, 2010).

Observa-se no estudo que menos de um décimo dos enfermeiros possuem especialização em oncologia, fato que chama a atenção, haja vista que o paciente oncológico necessita de profissionais capacitados para prestar assistência integral que alcance as suas reais necessidades. É essencial que tenham formação sobre a fisiopatologia do câncer como também sobre os aspectos psicológicos da oncologia, para que assim saibam lidar com os sofrimentos, angústias e sentimentos dos pacientes (ESTANQUE CM, 2011).

Além disso, os enfermeiros são os profissionais que estão em contato direto com os pacientes com câncer avançado, portanto, precisam ter uma visão ampliada sobre o conceito de paliação, para assim, poder desempenhar seu papel de educador e acolher a família e os doentes, proporcionando um cuidar que valorize o bem estar dos indivíduos. A enfermagem está em constante interação com os pacientes, haja vista, o maior tempo que passam com os pacientes, ocasionando muitas vezes desgaste emocional, por isso, é necessário que o enfermeiro esteja capacitado para trabalhar em oncologia integrando os cuidados paliativos (HERMES HR e LAMARCA ICA, 2013).

Sob este prisma, Fonseca A e Geovanini $F$ (2013), destacam que na graduação dos cursos na área da saúde a temática dos cuidados paliativos na maioria das vezes não é abordada nas disciplinas, dificultando assim, o trabalho dos profissionais. Observa-se uma presença minoritária de disciplinas referentes aos cuidados paliativo. Vale ressaltar que a medicina paliativa tornou-se uma área de especialização médica no Brasil apenas em 2011 (MACÊDO JALJ, 2015).

A formação dos profissionais de medicina está voltada para o tratamento e o diagnóstico das doenças e já no cuidado paliativo o foco é o paciente, que está além dos aspectos físicos, envolvendo aspectos psicológicos, sociais e espirituais, sendo necessário que a equipe médica se integre aos demais profissionais de saúde para tornar o cuidar integral e interdisciplinar (HERMES HR e LAMARCA ICA, 2013).

Os profissionais destacam o padrão cultural e a formação curativista como fatores que influenciam na integração dos cuidados paliativos aos pacientes oncológicos. No caso em questão, o padrão cultural está voltado para uma cultura em que a cura e a morte estão em disparidades, ocasionando um distanciamento da paliação, o que interfere na prática clínica do profissional de saúde (CRUZ ACVG, 2013).

Além desses fatores, foram ressaltados pelos profissionais o medo e a pressão familiar como pontos que interferem na integração da paliação aos outros cuidados oncológicos. O desconhecimento da família e do paciente a respeito dos cuidados paliativos e as influências culturais, sociais, religiosas e éticas acabam interferindo na decisão médica, sendo essencial a participação de uma equipe multiprofissional para ajudar a refletir sobre paliação e os benefícios quando inserida precocemente (OLIVEIRA RA, 2008).

Um ponto positivo do presente estudo está relacionado com a média de dias entre o encaminhamento e atendimento ao paciente oncológico pelos médicos paliativistas. Esse fato deve-se a implantação do NAT ONCO (Núcleo de Acolhimento e Triagem do paciente oncológico do IMIP) inaugurado em abril de 2016, o qual tem proporcionado $\mathrm{o}$ atendimento aos cuidados paliativos mais cedo aos pacientes com câncer metastático, como também ao papel da oncogeriatria da instituição que colabora com o atendimento de pacientes idosos com câncer avançado.

A inserção dos cuidados paliativos precocemente contribui para o aumento da sobrevida dos pacientes e melhora na qualidade de vida, sendo essencial que os profissionais passem a avaliar os pacientes e encaminhar para os paliativos de forma concomitante com outras terapêuticas. Sendo integrado como essencial para um cuidar que permita atender a singularidade de cada ser humano, acolher suas necessidades e proporcionar bem estar aos pacientes (CONNOR SR e BERMEDO MCS, 2014).

A abordagem precoce permite avaliar as necessidades dos pacientes, identificando sintomas que podem ser tratados, como exemplo, a dor do paciente oncológico, que pode ser minimizada por médicos paliativistas proporcionando aos pacientes conforto e bem estar (PINHEIRO TRSP, 2010). 
Nessa perspectiva, o desenvolvimento de pesquisas e ações relacionadas ao cuidado paliativo precoce, contribuirá para o aumento do conhecimento dos profissionais e, consequentemente, a uma prática eficaz.

Sendo assim, o estudo contribuiu por apresentar um panorama do conhecimento dos profissionais que trabalham com pacientes oncológicos na instituição. Sendo importante refletir e repensar sobre as práticas para auxiliar a integração dos cuidados paliativos no cotidiano deste setor.

\section{CONSIDERAÇÕES FINAIS}

O estudo demonstra que a maioria dos profissionais possui conhecimento e atitudes favoráveis ao cuidado paliativo, porém na prática ainda encontram dificuldades para integrá-lo concomitante com outras terapêuticas, devido à influência de fatores sociais e culturais que estão envolvidos na construção destes cuidados na sociedade. Observa-se que durante a formação acadêmica os profissionais não tiveram uma abordagem suficiente relacionada à temática dos cuidados paliativos, sendo necessária a inserção deste conhecimento na graduação para que possam fornecer uma assistência eficiente. Nessa perspectiva, a instituição por ser formadora de conhecimento pode, através desse estudo, respaldar a formulação de estratégias mediante as necessidades dos profissionais, contribuindo para o fortalecimento dos cuidados paliativos ao paciente oncológico.

\section{REFERÊNCIAS}

1. ATTY ATM, TOMAZELLI JG. Cuidados paliativos na atenção domiciliar para pacientes oncológicos no Brasil. Saúde debate, 2018; 42 (116): 223-9.

2. CARVALHO RT, PARSONS HA. Manual de Cuidados Paliativos. Rio de Janeiro: $2^{\underline{a}}$ ed. Ampliada e atualizada, 2012.

3. CHAVES JHB, et al. Cuidados paliativos na prática médica: contexto bioético. Revista Dor, 2011;12(3):250-5.

4. CONNOR SR, BERMEDO MCS. Worldwide Palliative Care Alliance, World Health Organization. Global Atlas of Palliative Care at the End of Life. 2014.

5. CRUZ ACVG. Cuidados paliativos em Portugal: a realidade do instituto português de oncologia do Porto. Dissertação (Mestrado em Medicina) - Instituto de Ciências Biomédicas Abel Salazar. Universidade do Porto, Portugal, 2013; 36 p.

6. ESTANQUE CM. A prática de cuidar o doente oncológico em fim de vida uma abordagem na perspectiva dos enfermeiros. Dissertação (Mestrado em Cuidados Paliativos) - Faculdade de Medicina. Universidade de Lisboa, Lisboa, 2011; 278 p.

7. FERREL BR, et al. Integration of palliative care into standard oncology care: American Society of Clinical Oncology Clinical practice guideline update. J Clin Oncol, 2016; 35 (1):96-112.

8. FIGUEIREDO MTA. Reflexões sobre os Cuidados Paliativos no Brasil. Revista Prática Hospitalar, 2006;8(47):36-40.

9. FONSECA A, GEOVANINI F. Cuidados Paliativos na Formação do Profissional da Área de Saúde. Revista Brasileira de Educação Médica, 2013;37(1):120-125.

10. HERMES HR, LAMARCA CA. Cuidados paliativos: uma abordagem a partir das categorias profissionais de saúde. Ciência Saúde Coletiva, 2013;18(9):2577-2588.

11. HUI D, et al. Availability and integration of palliative care at US cancer centers. Jama, 2010; 303 (11):1054-61.

12. HOCHMANB, et al. Desenhos de pesquisa. Acta Cir Bras. 2005;20(2): 02-9.

13. KASSA H, et al. Assessment of knowledge, attitude and practice and associated factors towards palliative care among nurses working in selected hospitals, Addis Ababa, Ethiopia. BMC Palliat Care, 2014;13(6):10-18.

14. OLIVEIRA RA. Cuidado Paliativo - Manual do Instituto Reinaldo Ayer de Oliveira. São Paulo: Conselho Regional de Medicina do Estado de São Paulo - CREMESP, 2008. 689 p.

15. PINHEIRO TRSP. Avaliação do grau de conhecimento sobre cuidados paliativos e dor dos estudantes de medicina do quinto e sexto anos. O Mundo da Saúde, 2010;34(3):320-326.

16. SILVA AF, et al. Cuidados paliativos em oncologia pediátrica: percepções, saberes e práticas na perspectiva da equipe multiprofissional. Revista Gaúcha Enfermgem, 2015; 36(2): 56-62.

17. SILVA C, et al. Estudo sobre Conhecimento, Atitudes e Práticas (CAP) relacionadas ao HIV-SIDA Junto dos funcionários do MJD. Manual - Ministério da juventude e desportos. República de Moçambique, 2010.

18. SILVA RCF, HORTALE VA. Cuidados paliativos oncológicos: elementos para o debate de diretrizes nesta área. Cad Saúde Pública, 2006; 22(10):2055-66.

19. World Health Organization. Noncommunicable diseases and their risk factors - Palliative Care [internet]. Disponível em: http://www.who.int/ncds/management/palliative-care/en/. Acesso em: 10 nov. 2018. 